\title{
Differential regulation of CCL21 in lymphoid/ nonlymphoid tissues for effectively attracting $T$ cells to peripheral tissues
}

\author{
James C. Lo, ${ }^{1}$ Robert K. Chin, ${ }^{1}$ Youjin Lee, ${ }^{2}$ Hyung-Sik Kang, ${ }^{2}$ Yang Wang, ${ }^{2}$ \\ Joel V. Weinstock, ${ }^{3}$ Theresa Banks, ${ }^{4}$ Carl F. Ware, ${ }^{4}$ Guido Franzoso, ${ }^{1}$ and Yang-Xin Fu ${ }^{1,2}$ \\ ${ }^{1}$ Committee on Immunology, and \\ ${ }^{2}$ Department of Pathology, University of Chicago, Chicago, Illinois, USA \\ ${ }^{3}$ Department of Medicine, University of Iowa, Iowa City, Iowa, USA \\ ${ }^{4}$ Division of Molecular Immunology, La Jolla Institute for Allergy and Immunology, San Diego, California, USA
}

\begin{abstract}
CC chemokine ligand 21 (CCL21)/secondary lymphoid chemokine (SLC), a ligand for CC chemokine receptor 7 (CCR7), has been demonstrated to play a vital role in the homing and localization of immune cells to lymphoid tissues, but its role in nonlymphoid tissues largely remains undefined. Here, we provide evidence that CCL21 in lymphoid and nonlymphoid tissues is differentially regulated by lymphotoxin-dependent (LT-dependent) and -independent mechanisms, respectively. This differential regulation is due to the selective regulation of the CCL21-Ser/CCL21a but not the CCL21$\mathrm{Leu} / \mathrm{CCL} 21 \mathrm{~b}$ gene by the LT and noncanonical NF- $\mathrm{KB}$ pathways. This alternate pathway, not dependent on LT or lymphocytes, leading to constitutive expression of CCL21 in nonlymphoid tissues, is critical for the initial recruitment of $\mathrm{T}$ lymphocytes to peripheral effector sites. CCL21 expression is subsequently further enhanced in a LT-dependent fashion following airway challenge, potentially facilitating a positive feedback loop to attract additional CCR7 ${ }^{+}$effector cells. These findings establish an essential role for CCL21 in the recruitment of effector T cells to peripheral tissues and suggest that LT-dependent and -independent regulation of CCL21 plays a role in balancing the central and peripheral immune responses between lymphoid and nonlymphoid tissues.
\end{abstract}

J. Clin. Invest. 112:1495-1505 (2003). doi:10.1172/JCI200319188.

\section{Introduction}

The high degree of specificity and complexity demanded in the immune system is achieved in part by the delicate compartmentalization and efficient organization of its cellular constituents. Recent studies have identified members of the TNF superfamily as critical regulators in the organization of the immune system $(1,2)$. Lymphotoxin (LT), in particular, is required for the formation of lymph nodes and Peyer's patches (PPs), as well as the development of follicular DC networks, germinal centers, and effective $\mathrm{T} / \mathrm{B}$ lymphocyte segregation in the spleen (1). Although LT is found in two distinct forms (soluble

Received for publication June 12, 2003, and accepted in revised form September 2, 2003.

Address correspondence to: Yang-Xin Fu, Department of Pathology, University of Chicago, 5841 South Maryland Avenue J-541, MC3083, Chicago, Illinois 60637, USA.

Phone: (773) 702-0929; Fax: (773) 834-8940;

E-mail: yfu@midway.uchicago.edu.

Conflict of interest: The authors have declared that no conflict of interest exists.

Nonstandard abbreviations used: lymphotoxin (LT); Peyer's patches (PP); i.p., intraperitoneally; OVA-specific T cell (OTII); CC chemokine receptor (CCR); CC chemokine ligand (CCL); ERBI-1 ligand chemokine (ELC); Cys-X-Cys chemokine receptor (CXCR); Cys-X-Cys cxhemokine ligand (CXCL); B-lymphocyte chemoattractant (BLC); secondary lymphoid tissue chemokine (SLC); phycoerythrin (PE); T cell receptor (TCR); soluble egg antigen (SEA); IFN- $\gamma$-induced protein-10 (IP-10); stromal cell-derived factor (SDF); rat insulin promoter (RIP).
$L T \alpha_{3}$ and membrane-bound $L T \alpha_{1} \beta_{2}$ ), most of the phenotypes observed in $L T \alpha^{/-}$mice are attributed to the lack of membrane LT rather than soluble LT signaling, since defects in $L T \beta^{-/-}$and $L T \beta R^{-/-}$mice closely mirror those seen in $L T \alpha^{-/}$mice (1). One of the major signaling pathways activated by the LT $\beta R$ is the NF- $\mathrm{KB} /$ Rel family of transcription factors (3). Consistent with a major role for NF- $\kappa B$ in transducing LT $\beta R$ signals, several single-gene knockout mice for NF- $\kappa B$ transcription factors (RelA, RelB, and p52) or NF- $\kappa B$ regulatory proteins (I $\kappa B$ kinase $\alpha$, IKK $\alpha$; $\mathrm{NF}-\kappa \mathrm{B}$-inducing kinase, NIK) have produced phenotypes similar to LT-deficient mice (4-7).

Chemokines and chemokine receptors support cell migration and homing to lymphoid tissues (8). T lymphocyte entry to LNs requires CC chemokine receptor 7 (CCR7) and its ligands CCL19 (EBI-1 ligand chemokine, ELC) and CCL21 $(9,10)$. In contrast, B lymphocyte homing to PPs is more complex with major contributions by Cys-X-Cys receptor 4 (CXCR4) (receptor for Cys-X-Cys ligand 12, CXCL12/ SDF-1 $\alpha$ ), CXCR5 (receptor for CXCL13/B-lymphocyte chemoattractant, BLC), and CCR7 (11). Murine CCL21, intriguingly called secondary lymphoid tissue chemokine (SLC), is expressed in both lymphoid and nonlymphoid tissues and encoded by two to three (depending on strain) genes that are distinguished by a single nucleotide change leading to a leucine and serine discrepancy at position $65(12,13)$. 
This single residue change has no apparent effect on chemotactic activity, since the two forms of CCL21 are each capable of attracting lymphocytes and inducing lymphoid neogenesis when ectopically expressed in the pancreas (14).

Organ or target localization adds to the specificity of the immune response and is critical for pathogen clearance at the site of infection. Whereas significant advances have been made on the chemokine requirements for $\mathrm{T}$ and $\mathrm{B}$ cell entry to lymphoid tissues, much less is known about how lymphoid tissue chemokines regulate lymphocyte entry to nonlymphoid tissues $(11,15)$. This trafficking step is especially pertinent because nonlymphoid tissues typically represent the infected/effector sites. Specific chemokines are likely required for lymphocyte homing to nonlymphoid tissues and potentially impart unique molecular fingerprints on different nonlymphoid tissues to achieve tissue-specific migration of lymphocytes in natural defense and autoimmunelike situations $(15,16)$.

Membrane LT is essential for the expression of various lymphoid tissue chemokines that allow homing of immune cells to the spleen (17-19). However, the strong inflammatory infiltrates observed in the lungs of $L T \alpha^{-1-}$ mice to environmental antigens and delivered antigens even in the absence of draining lymph nodes prompted us to examine the potential differential regulation of CCL21 expression in nonlymphoid tissues. We have found that in contrast to the spleen, where CCL21-Ser is dominant and LT dependent, CCL21-Leu is preferentially expressed in the lung in a LT-independent fashion. However, under inflammatory conditions LT-mediated induction of CCL21-Ser serves to enhance the local chemokine gradient. More importantly, disruption of the CCL21/CCR7 chemokine and receptor axis severely impaired $T$ cell trafficking to the lung, revealing the essential role of CCL2 1 in T cell homing to the lung. The differential regulation of CCL21 expression in the lung and spleen suggests a delicate chemokine balance between lymphoid and nonlymphoid tissues coordinates the central and peripheral immune responses.

\section{Methods}

Mice. $L T \alpha^{-/}$and $L T \beta R^{-/-}$mice were backcrossed to B6 mice and maintained under specific pathogen-free conditions as described $(20,21)$. LT $\beta^{-/-}$mice were from Taconic, Germantown, New York, USA. B6 and Rag-1 $1^{-/-}$mice were from The Jackson Laboratory (Bar Harbor, Maine, USA). Animal care and use were in accordance with institutional and National Institutes of Health guidelines.

Flow cytometric analysis and cell isolation. Phycoerythrin-conjugated (PE-conjungated) CD11c, Thy1.2, B220, CD62L, T cell receptor (TCR) Va2; FITC-conjugated I-A ${ }^{\mathrm{b}}$, NK1.1, cychrome-conjugated CD4; and biotin-conjugated VCAM-1, ICAM-1, DX5, Thy1.1 antibodies were from PharMingen, San Diego, Cali- fornia, USA. To detect CCR7 expression, ELC-Fc or human $\mathrm{Fc}$ binding was followed by staining with FITC-conjugated donkey anti-human IgG (Jackson ImmunoResearch Laboratories Inc., West Grove, Pennsylvania, USA). Lung tissues were digested three times by shaking for 30 minutes at $37^{\circ} \mathrm{C}$ in RPMI 1640 medium containing $1 \mathrm{mg} / \mathrm{ml}$ collagenase VIII (Sigma-Aldrich, St. Louis, Missouri, USA) and 2\% FBS (Invitrogen Corp., Carlsbad, California, USA). Lung cells were passed through a Nytex filter (Becton Dickinson, Franklin Lakes, New Jersey, USA), erythrocytes were lysed with an ammonium, chloride, potassium (ACK) lysis buffer, and the total number of cells was enumerated by trypan blue exclusion. The total number of CD4, B, dendritic, and NK cells within the lung was then determined by flow cytometry. CD4 and B220 antibodies were used to identify $\mathrm{CD}^{+} \mathrm{T}$ cells and B cells. DCs were identified as cells that double stained with CD11 $\mathrm{c}$ and $\mathrm{I}-\mathrm{A}^{\mathrm{b}}$ antibodies. NK cells were identified by double staining with NK1.1 and DX5 antibodies. Splenic and lung stromal cells were enriched from collagenase-treated tissues after removal of adherent fibroblasts. Stromal cells were cultured with media in the presence of either $2 \mu \mathrm{g} / \mathrm{ml}$ anti-LT $\beta$ R (AF.H6) or control hamster antibody (Ha4/8) for 48 hours. Cells were stained and analyzed by flow cytometry on a FACScan or FACScalibur using CellQuest (BD Biosciences, San Jose, California, USA) or FlowJo (Tree Star, Ashland, Oregon, USA) software.

Gene array. Chemokine gene array was performed according to the manufacturer's instructions (SuperArray Inc., Bethesda, Maryland, USA). Total RNA was isolated using TRI reagent (Sigma-Aldrich). Lymph nodes were removed from lung tissues prior to RNA extraction.

Immunohistochemistry. Frozen sections of spleen were prepared, fixed, and stained as previously described (22). Formalin-fixed lung tissues were sectioned and stained for CCL21 and counterstained by hematoxylin. Biotin-conjugated CCL21 (R\&D Systems Inc., Minneapolis, Minnesota, USA) and FITC-conjugated B220 (PharMingen) antibodies were used.

ELISA for CCL21. Splenic and lung homogenates were prepared and assayed for CCL21 as described (22). Recombinant CCL21, CCL21 antibodies, and biotin-conjugated CCL21 antibodies were from R\&D Systems, Inc. Lymph nodes were removed prior to preparation of tissue homogenates. The amount of CCL21 was determined from the standard curve and normalized by tissue weight.

Soluble egg antigen airway challenge and adoptive transfer. B6 mice were challenged with soluble egg antigen (SEA) as previously described (23). Mice were sensitized on day 0 with 2,500 irradiated Schistosoma mansoni eggs i.p., challenged with $5 \mu \mathrm{g}$ of SEA by intratracheal injection or aspiration on day 7, and killed on day 11. For LT $\beta$ neutralization studies, $150 \mu \mathrm{g}$ doses of anti-LT $\beta$ antibodies (BB.F6) or control hamster Ig (Ha4/8) were injected intraperitoneally (i.p.) on days 7 and 9. Lung leukocytes were prepared from SEA-challenged mice, 


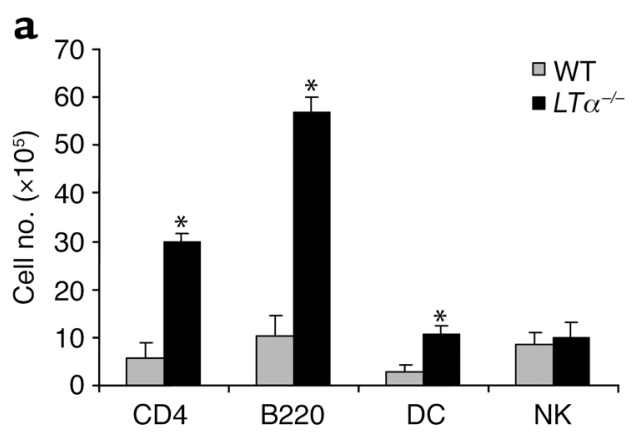

b
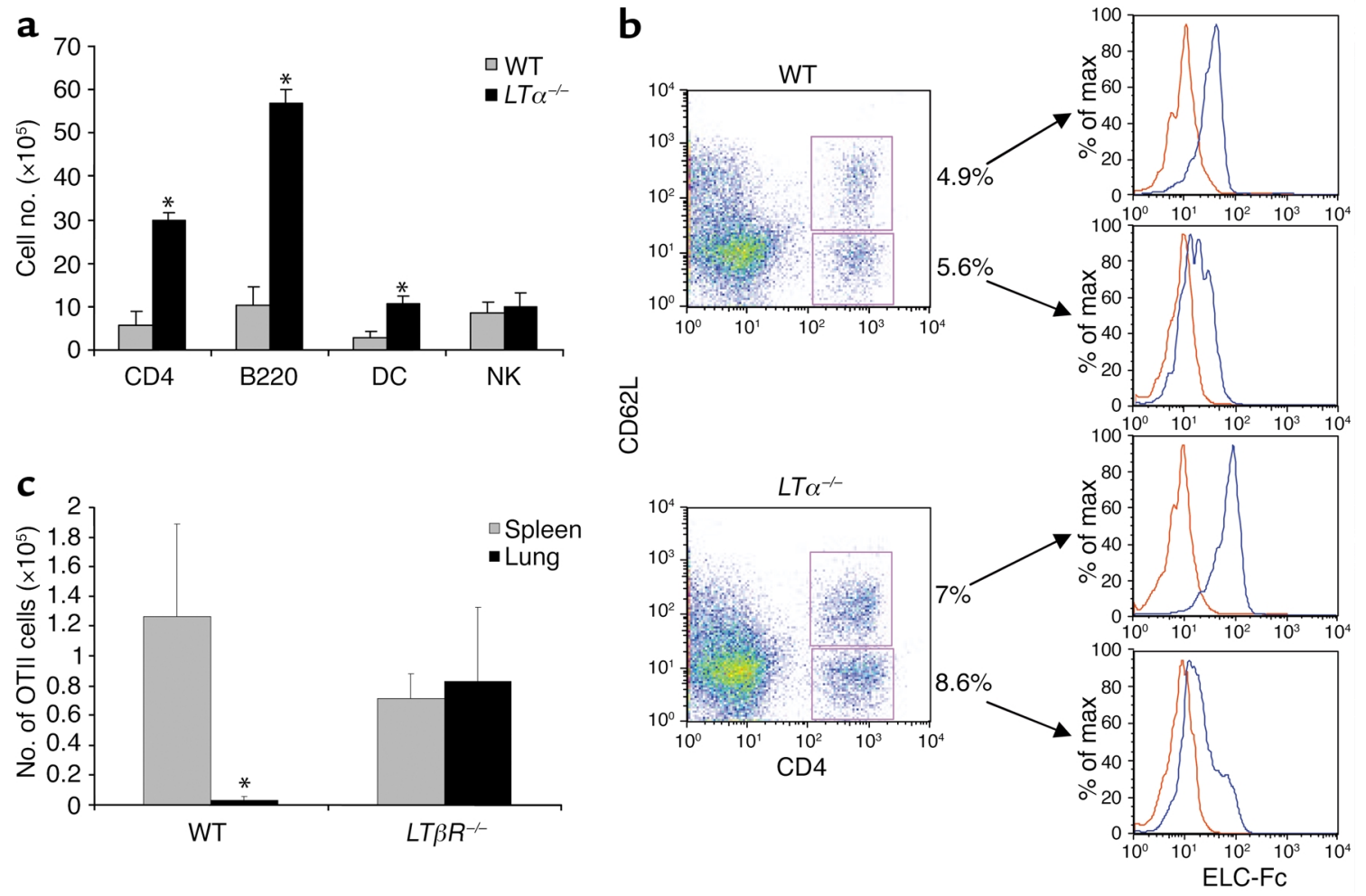

Figure 1

Leukocyte accumulation in lungs of LT-deficient mice. (a) Increased numbers of CD4 $4^{+}, \mathrm{B} 220^{+}$, and DCs in lungs of $L T \alpha^{-1-}$ mice. Data are mean \pm SD of triplicate samples and represent at least three independent experiments. (b) CCR7 expression on naive and activated phenotype pulmonary $C D 4^{+} T$ cells. Representative dot plots and histograms of CCR7 chemokine expression using ELC-Fc (blue lines) compared to control human- $\mathrm{Fc}$ (red lines) on $\mathrm{CD} 4^{+} \mathrm{CD} 62 \mathrm{~L}^{\text {high }}$ and $\mathrm{CD} 4^{+} \mathrm{CD} 62 \mathrm{~L}^{\text {low }}$ cells. (c) Altered homing of $\mathrm{CD} 4^{+} \mathrm{T}$ cells to the lungs of $L T \beta R^{-/-}$mice. The spleens and lungs of WT and $L T \beta R^{-1-}$ mice were analyzed for the presence of OTIl cells 2 days after adoptive transfer i.p. of Thy $1.1^{+}$OTII cells. Data are mean \pm SD of four or more mice per group. ${ }^{*} P<0.05$ between WT lung and WT spleen, and WT lung and $L T \beta R^{-/-}$lung as calculated by the Student's $t$ test. Max, maximum.

blocked with anti-FcR (2.4G2) and rat serum, labeled with Thy1.2-PE antibody and CFSE, and treated with CCL19 $(10 \mu \mathrm{g} / \mathrm{ml})$ or PBS for $45 \mathrm{~min}$. at $37^{\circ} \mathrm{C}$. Cells were transferred i.v. into $\mathrm{LTO}^{/ /}$recipients. To trace the transferred cells, cells were either prelabeled with Thy1.2-PE antibody or bore Thy1.1 or Ly5.1 congenic markers. The recipient mice were killed 1 hour later, bled, and perfused before collection of lung leukocytes. For in vivo neutralization of CCL21, 30- $\mu$ g doses of antiCCL21 or control goat Ig (Southern Biotechnology Associates Inc., Birmingham, Alabama, USA) were administered i.v. 1 hour prior to adoptive transfer, and the mice were killed 4 hours after transfer. For OTII (OVAspecific $T$ cell) transgenic transfer, Thy $1.1^{+}$OTII cells were incubated with $5 \mu \mathrm{g} / \mathrm{ml}$ OVA 323-339 peptide in vitro for 60 hours and then adoptively transferred i.p. to Thy $1.2^{+}$WT or $L T \beta R^{-/-}$mice. The recipient mice were killed 40 hours later, and the spleen and perfused lungs were analyzed for the presence of OTII cells.

Real-time PCR assay. Real-time PCR was performed as previously described $(14,22,24)$. Each cDNA sample was amplified in duplex for CCL21 and GAPDH with the TaqMan Universal PCR master mixture according to the manufacturer's instructions (PE Applied Biosystems, Foster City, California, USA). The concentration of the target gene was determined using the comparative $\mathrm{C}_{\mathrm{T}}$ (threshold cycle number at a cross-point between amplification plot and threshold) method and normalized to the internal GAPDH control.

In vivo gene expression. Fifty micrograms of anti-LT $\beta \mathrm{R}$ (3C8) or isotype control rat antibody (PharMingen) were injected i.p. into WT mice (25). Spleen and lung tissues were collected for mRNA isolation 8 hours later. cDNA was prepared and real-time PCR analysis for CCL21-Ser, CCL21-Leu, and GAPDH was performed.

\section{Results}

Preferential accumulation and homing of $T$ cells to the lungs of LT-deficient mice. Earlier gene targeting studies of the LT pathway revealed a spontaneous accumulation of immune cells in the lungs of $L T \alpha^{--}$and $L T \beta R^{-1-}$ mice, the basis of which remains unknown $(21,26)$. In order to further understand the nature of the inflammatory infiltrate in $L T \alpha^{--}$mice, we determined the cellular composition of immune cells in the lungs of WT and $L T \alpha^{-/-}$ mice (Figure 1a). Consistent three- to fivefold increases in the number of $\mathrm{CD}^{+}{ }^{+} \mathrm{T}$ cells, B cells, and DCs were detected in the lungs of $L T \alpha^{-/}$and $L T \beta R^{-/-}$(data not shown) compared to WT mice (Figure 1a). A more modest twofold increase in $\mathrm{CD}^{+} \mathrm{T}$ cells was also observed in 
$L T \alpha^{-1-}$ lungs (data not shown). The increase in $\mathrm{CD}^{+} \mathrm{T}$ cells, B cells, and DCs in $L T \alpha^{/-}$lungs is specific as NK cells and macrophages are not increased (Figure 1a; data not shown). Interestingly, the three cell types overrepresented in the $L T \alpha^{-1}$ lung are all known to express the CCR7 chemokine receptor and exhibit chemotactic activity towards its ligand CCL21 (27-31). This raises the possibility of altered CCL21 expression in the lung, leading to the accumulation of leukocytes in the lung.

The increased numbers of leukocytes in the lungs of LT-deficient mice may reflect a selective accumulation of naive or activated cells. To better characterize the nature of the lymphoaccumulation, flow cytometry analysis was performed to determine the activation status of the T lymphocytes in WT and $L T \alpha^{-1}$ lungs. The analysis revealed that the lymphoaccumulation includes both activated $\left(\mathrm{CD} 62 \mathrm{~L}^{\text {low }}\right)$ and naive $\left(\mathrm{CD} 62 \mathrm{~L}^{\text {high }}\right)$ phenotype $\mathrm{CD}^{+} \mathrm{T}$ cells in the lungs of $\mathrm{LTO}^{-/}$mice (Figure 1b). Staining with the activation marker CD44 also showed that both antigen-experienced and naive phenotype $\mathrm{CD} 4^{+} \mathrm{T}$ cells accumulate in the lungs of $L T \alpha^{-/}$mice. Additionally, we found a slight increase in activated B cells and mature DCs in the lungs of $L T \alpha^{-1-}$ mice (data not shown). To test our hypothesis that CCL21 expression in the lung was attracting CCR7 expressing T cells, ELC-Fc was used to determine if $\mathrm{CCR} 7^{+}$cells are present in the lung. We found that both

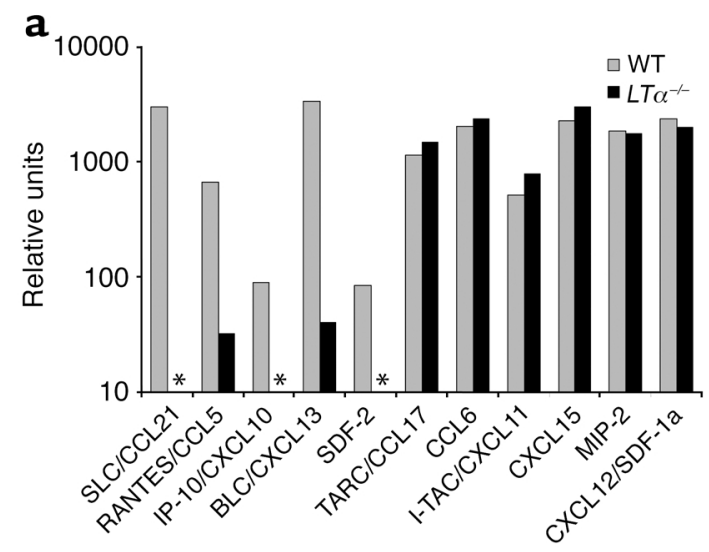

c.
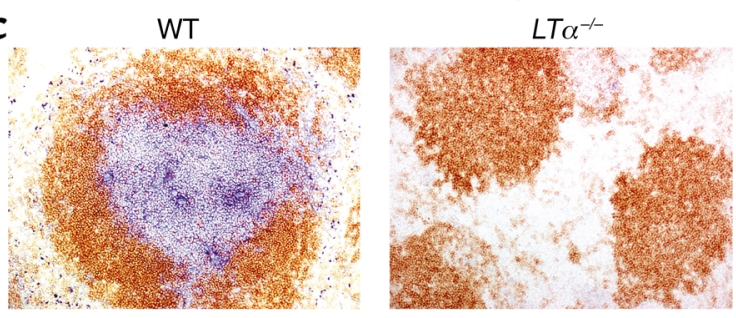

CCL21/B220 naive and activated phenotype $\mathrm{CD} 4^{+} \mathrm{T}$ cells express CCR7 (Figure 1b). These data indicate that both CCR7 ${ }^{+}$ activated or naive $T$ cells accumulate in lungs of LTdeficient mice and suggest that CCL21 may be responsible for attracting the cells via CCR7.

The lymphoaccumulation observed in the lungs of LTdeficient mice may be a result of enhanced homing to the lung or increased cell proliferation. To directly interrogate the role of altered homing, antigen-activated WT OTII CD4+ TCR transgenic T cells bearing the Thy 1.1 congenic marker were adoptively transferred into WT or $L T \beta R^{-/-}$mice expressing Thy1.2. The spleen and lung were analyzed for the presence of OTII cells 40 hours after adoptive transfer. CD4+ OTII cells preferentially homed to the spleen and significantly lower to the lung in WT mice $(P<0.05$ between WT spleen and lung using the Student's $t$ test) (Figure 1b). By contrast, the homing profile of $\mathrm{CD}^{+} \mathrm{OTII}$ cells was perturbed in LT-deficient mice with robust homing to the lung that was on par with the spleen and significantly greater than WT lung $(P<0.05$ between LT-deficient and WT lungs using the Student's $t$ test) (Figure 1b). Increased homing to the lungs of LT-deficient mice included naive OTII cells (data not shown). These results indicate that $\mathrm{CD} 4^{+} \mathrm{T}$ cells preferentially home to the lung in LT-deficient mice and suggest that increased homing is a major mechanism for the accumulation of leukocytes in the lung.
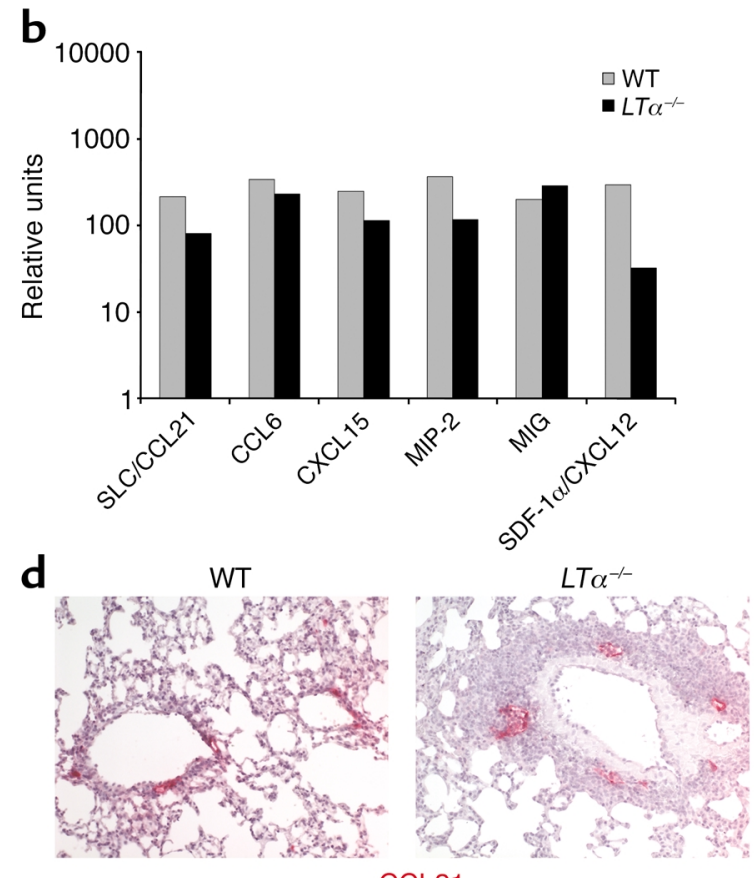

CCL21

Figure 2

Lymphotoxin regulates splenic chemokine expression. (a, b) RNA from WT and $L T \alpha^{-1-}$ spleen $(\mathbf{a})$ and lung (b) were subjected to chemokine gene array. All signals were normalized to $\beta$-actin, which was set to 10,000. Asterisks indicate that no signal above background was detected. RNA from at least three mice was pooled together. A representative blot from two independent experiments is shown. (c, d) LT regulates splenic but not pulmonary CCL21. OCT-embedded (Sakura Finetek, Torrance, California, USA) spleen (c) sections $(\times 100)$ were stained for CCL21 (blue) and B220 (brown). Formalin-fixed lung (d) sections $(\times 200)$ were stained for CCL21 (red) and counterstained with hematoxylin. A representative staining from more than five experiments is shown. TARC, thymus and activation-regulated chemokine; I-TAC, IFN-inducible T cell $\alpha$-chemoattractant; MIG, monokine induced by IFN- $\gamma$; MIP, macrophage inflammatory protein. 

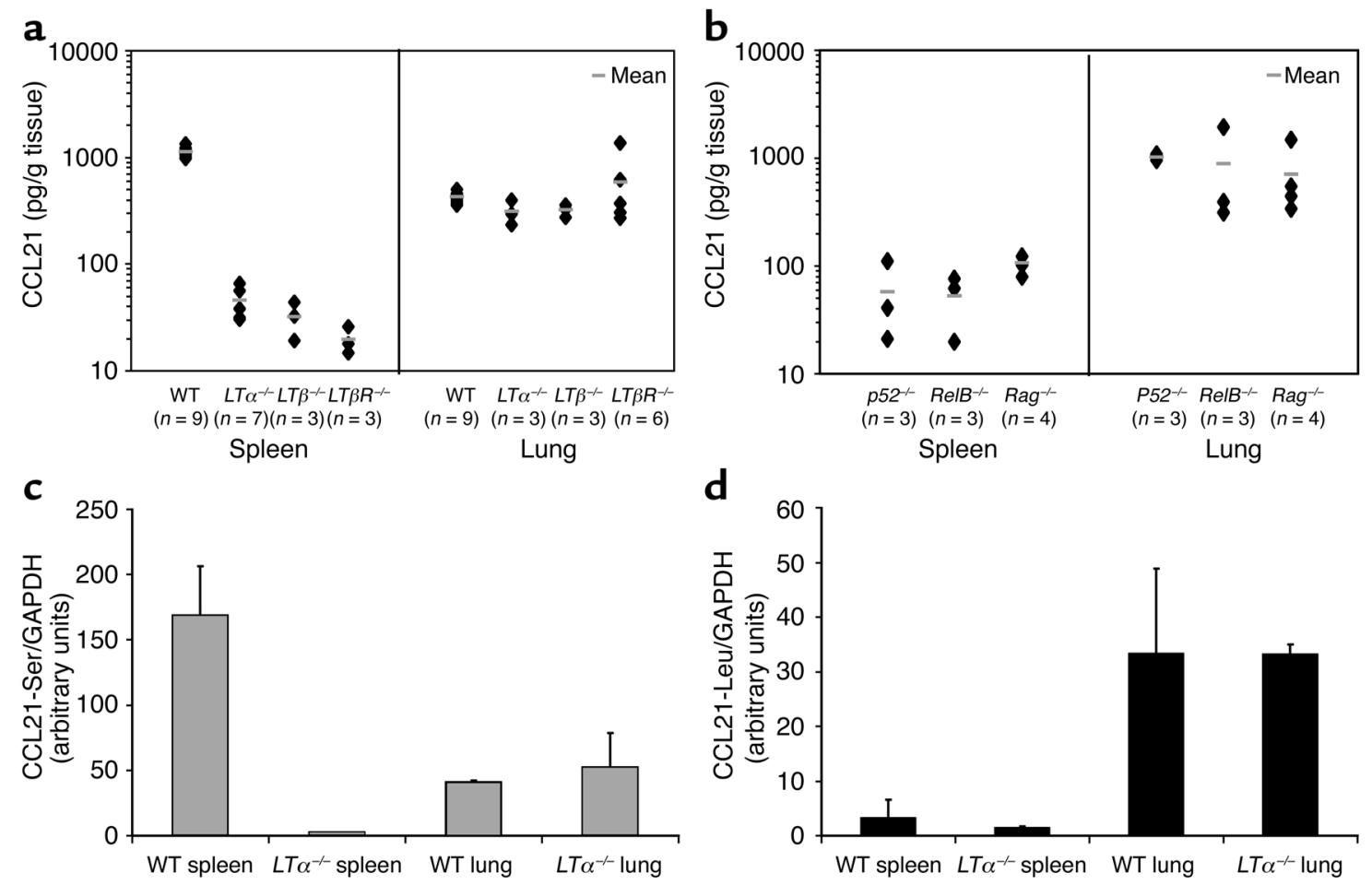

Figure 3

CCL21 is differentially regulated in the spleen and lung. (a) LT signals are essential for spleen but not lung CCL21. ELISA for CCL21 was performed on spleen and lung homogenates prepared from various LT-deficient mice. Each sample was normalized by tissue weight. (b) Differential requirements for NF-KB and lymphocytes in the expression of CCL21 in the spleen and lung. ELISA for splenic and lung CCL21 from various NF- $\mathrm{KB}-\mathrm{defi}-$ cient and $\mathrm{Rag}^{-1-}$ mice, calculated as in a. Each symbol represents data from a single mouse. (c, d) LT selectively regulates the expression of CCL21Ser but not CCL21-Leu. Real-time PCR analysis was performed on the spleen and lung of WT and $L T \alpha^{-1-}$ mice with conditions specific for CCL21-Ser (c) and CCL21-Leu (d). The columns and bars represent the mean \pm SD.

Lymphotoxin regulates splenic chemokine expression. We hypothesized that increased expression of chemokines in the lung or an altered balance between the spleen and lung of LT-deficient mice was responsible for enhanced cell homing to the lung. To unravel the relationship between LT and chemokines in a systematic and unbiased fashion, we studied chemokine expression in LTdeficient mice using gene array analysis. Splenic and lung RNA from naive WT and $L T \alpha^{-/-}$mice were extracted and subjected to a chemokine gene array. Our analysis revealed dramatic reductions in the chemokines SLC/CCL21, RANTES, IFN- $\gamma$-inducible-10 (IP-10)/ CXCL10, BLC/CXCL13, and stromal cell-derived factor-2 (SDF-2) in the spleens of $L T \alpha^{/-}$mice (Figure 2a). The expression of numerous other chemokines, including thymus and activation-regulated chemokine/ CCL17, CCL6, interferon inducible-T cell alpha chemoattractant/CXCL11, CXCL15, macrophage inflammatory protein- 2 , and SDF- $1 \alpha /$ CXCL12 was comparable between WT and $L T \alpha^{-1}$ spleens (Figure 2a). Our analysis of the lung revealed similar chemokine profiles between WT and $L T \alpha^{-/}$mice and, importantly, that none of the chemokines were significantly elevated in LT-deficient mice (Figure 2b). These data indicate that $\mathrm{LT}$ is required for the basal expression of a specific subset of chemokines in the spleen but not in the lung.
An alternate hypothesis was that the distorted localization of chemokines or imbalanced chemokine gradient between the spleen and lung in LT-deficient mice is responsible for the perturbed homing. The chemokine CCL21 piqued our interest because genetic studies examining $L T \alpha^{-/}$, plt/plt (both with dramatically reduced lymphoid tissue CCL21) and CCR $7^{-/-}$(CCL21 receptor) mice revealed a critical role for CCL21 in T cell homing to lymphoid organs $(9,10)$. Additionally, plt/plt or WT mice treated with anti-CCL21 antibodies paradoxically mounted a delayed but exaggerated peripheral immune response $(32,33)$. To visualize the expression pattern of CCL21 in the tissues, we performed immunohistochemistry staining for CCL21 on the spleens and lungs of WT and $L T \alpha^{-/-}$mice. CCL21 staining was noticeably absent in the $L T \alpha^{/-}$spleen, in contrast to the abundant staining seen in the $\mathrm{T}$ cell zones of WT spleen (Figure 2c). LT deficiency, however, had no effect on the pattern of CCL21 staining in the lung (Figure 2d). The immunohistochemistry staining confirmed the essential role of LT for CCL21 expression in the spleen but not in the lung.

Baseline CCL21 expression in the lung is independent of $L T$, lymphocytes, p52, and RelB. We propose that LT may not positively regulate CCL21, a CCR7 ligand, in the lung and that $L T \alpha^{/-}$mice may have similar or increased lev- 
a

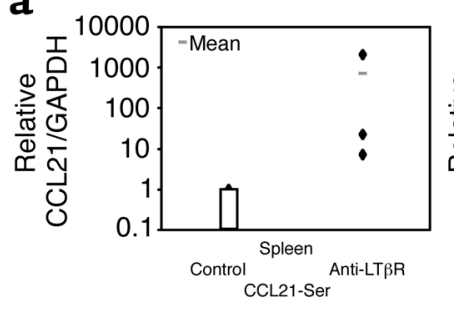

C

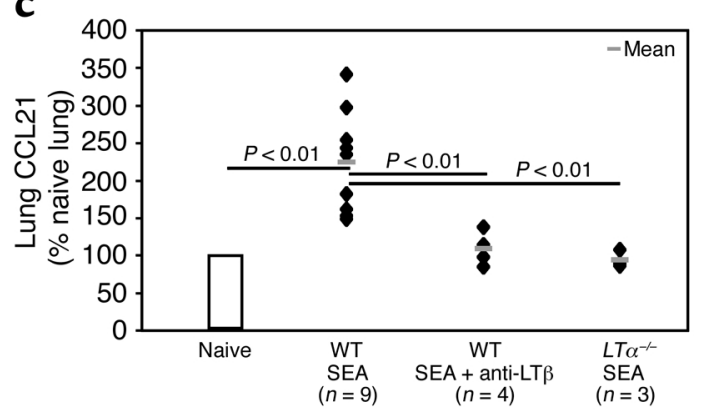

b
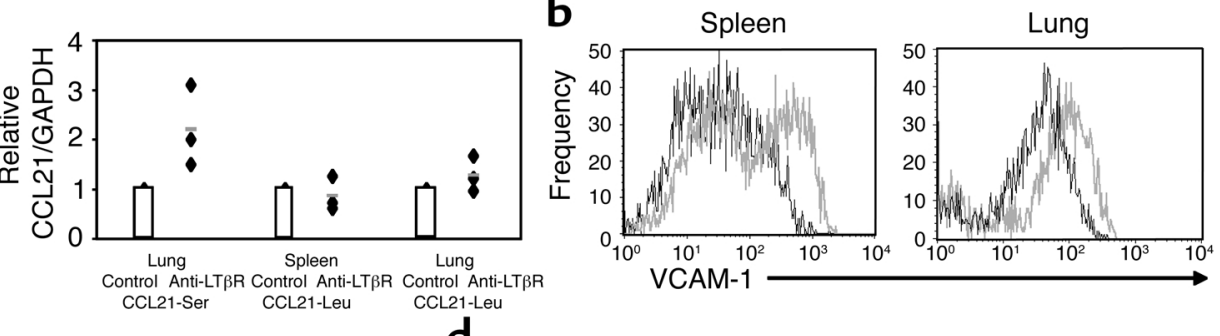

d

\section{Figure 4}

Airway inflammation induces the CCL21-Ser gene in an LT-dependent fashion. (a) LT $3 R$ signaling specifically induces CCL21-Ser. WT mice were injected i.p. with control or agonistic LT $\beta R$ antibodies. RNA from the spleens and lungs were analyzed by real-time PCR for CCL21-Ser and CCL21-Leu. Each point reflects the fold induction in CCL21. (b) Spleen and lung cells respond to LT $\beta R$ signals. Representative histograms of stromal cells from the spleen or lung stimulated with species control (black) or agonistic anti-LT $\beta R$ (gray) antibody and stained with VCAM-1 antibody for analysis by flow cytometry is shown. (c) Airway inflammation induction of CCL21 in the lung is LT-dependent. WT or $L T \alpha^{-1-}$ mice were challenged with SEA and control or anti-LT $\beta$ antibodies were administered i.p. during the challenge phase only. Mice were killed 3 to 4 days after challenge and pulmonary CCL21 quantified as previously described (see Figure 3a). Student's $t$ tests were performed between naive and challenged, control challenged and anti-LT $\beta$-challenged, and WT and $L T \alpha^{-/}$SEA-challenged groups and the resultant $P$ values are shown. (d) Airway inflammation specifically induces the CCL21-Ser gene. Real-time PCR for the two CCL21 genes was performed on RNA extracted from lungs of naive and SEA-challenged WT and $L T \alpha^{-/}-$mice. PCR reactions were performed in duplex with a $\mathrm{GAPDH}$ internal control for normalization. Columns and bars represent the mean $\pm \mathrm{SD}$.

els of CCL21 in the lung that is responsible for the accumulation of T cells, B cells, and DCs. To test this hypothesis, we performed an ELISA for CCL21 from splenic and lung homogenates of WT and various LTdeficient mice for a more quantitative assessment of actual protein levels (Figure 3a). In the spleen, $L T \alpha^{-/}$ mice displayed more than a 20 -fold reduction in CCL21 (Figure 3a). Since $L T \alpha^{-/-}$lacks both soluble and membrane forms of LT, we examined $L T \beta^{-/-}$to dissect the contribution of membrane LT signals. $L T \beta^{-/}$mice exhibited a similar defect in CCL21 expression in the spleen but not in the lung (Figure 3a). To define whether LT $\beta R$ is essential for the expression of CCL21 in the spleen and lungs, we analyzed $L T \beta R^{-/-}$mice. Similar to $L T \alpha^{/-}$and $L T \beta^{-/-}$mice, $L T \beta R^{-/-}$mice have very low levels of CCL21 in the spleen, confirming that signaling of LT $\beta R$ by membrane $L T$ is required for proper expression of CCL21. However, no significant reduction of CCL21 in the lung was found in the LT-ligand and receptor-deficient mice, leading to a reversal of the WT situation with relatively high levels of CCL21 in the lungs of LT-deficient mice. The data demonstrate that, in stark contrast to the spleen, LT is not required for the constitutive expression of CCL21 in the lung.

Recent reports have shown that LT utilizes the noncanonical p52/RelB NF- $\mathrm{KB}$ pathway to induce chemokine expression $(3,25)$. To exclude the possibil- ity that other ligands or factors in the lung may be compensating for LT to activate p52 and RelB, we quantified CCL21 expression in the lungs and spleens of $p 52^{-/-}$and $\mathrm{RelB}^{-/-}$mice (Figure $3 \mathrm{~b}$ ). Our analysis again revealed a similar segregation between the spleen and lung: that $p 52^{-/-}$and $\mathrm{RelB}^{-/-}$mice had approximately a 10 -fold reduction in CCL21 in the spleen without appreciable loss in the lung (Figure 3b). Finally, we tested for the requirement of lymphocyte-derived factors in the maintenance of CCL21 in the lung (Figure 3b). Although lymphocyte-derived factors are essential for splenic CCL21 expression, lymphocytes are not required for the expression of CCL21 in the lung because WT and $\mathrm{Rag}^{-1-}$ mice had similar levels of pulmonary CCL21 (Figure 3b). These results indicate that constitutive expression of CCL21 in nonlymphoid tissues such as the lung is independent of lymphocytes, and the LT and p52/RelB pathways.

Lymphotoxin selectively regulates CCL21-Ser. B6 mice express two different CCL21 genes, but the role of LT in the regulation of each of these genes has not been well defined. The abundant expression of CCL21 in nonlymphoid tissues of $L T \alpha^{-1}$ mice suggested to us that an LT-independent CCL21 gene is predominantly expressed in nonlymphoid tissues. To formally address this issue, real-time PCR analysis with probes specific for each of the two CCL21 genes was performed on 
a

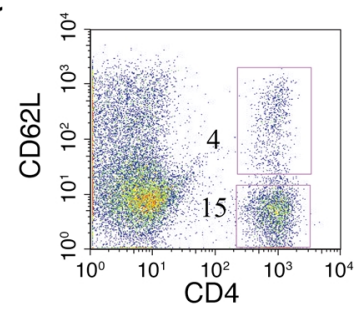

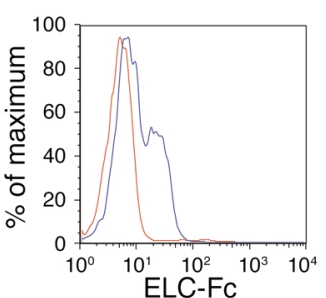

b

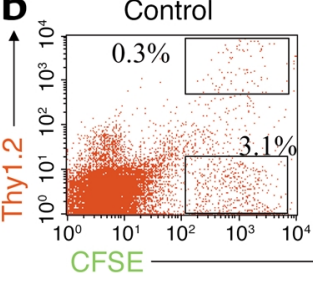

CCR7-desensitized Pretransfer

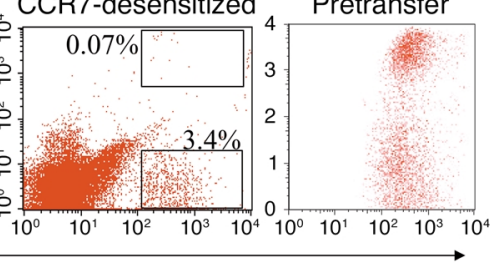

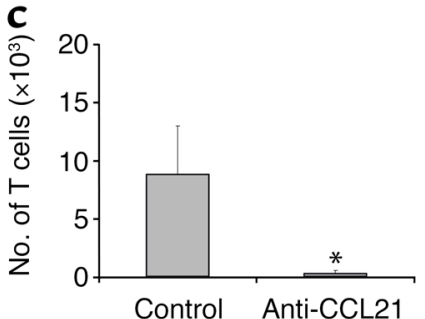

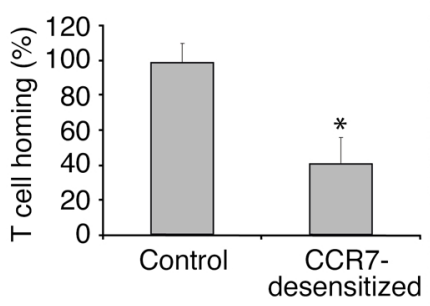

\section{Figure 5}

CCL21 and CCR7 on the T cell are required for homing to the lung. (a) Phenotypic characterization of T cells from lungs of SEA-challenged mice used in adoptive transfer experiments. Representative staining for CD4 and CD62 L is shown. The numbers adjacent to the gates indicate the percentage of cells within the gates. A representative histogram is shown for CCR7 expression on gated CD4 ${ }^{+} \mathrm{T}$ cells using control human-Fc (red) and ELC-Fc (blue). (b) The CCR7 chemokine receptor on the T cell is required for homing to the lung. Lung cells from SEAchallenged mice were prelabeled with Thy1.2-PE and CFSE, treated with PBS or CCL19 to desensitize the CCR7 receptor, and adoptively transferred i.v. to naive $L T \alpha^{/-}$mice. Recipient mice were killed 1 hour later, and the lungs were collected for analysis. Leukocytes were isolated from the lungs and subjected to flow cytometry analysis. Representative dot plots from control, CCR7 desensitized groups, and cells prior to transfer are shown. The numbers next to the gates indicate the percentage of cells within the gate. (c) CCL21 is required for T cell homing to the lung. Recipient mice were pretreated 1 hour prior to adoptive transfer with anti-CCL21 or control antibodies. Lung leukocytes were extracted 4 hours after adoptive transfer, and the T cells were enumerated. ${ }^{*} P<0.05$ as calculated from Student's $t$ test. Data are pooled from at least four independent experiments.

RNA extracted from the spleens and lungs of WT and LT-deficient mice. The abundant CCL21-Ser transcripts in the WT spleens were conspicuously absent in the $L T \alpha^{-1-}$ spleens (Figure $3 \mathrm{c}$ ). In contrast, the absence of LT did not alter the transcriptional activity of CCL21Ser in the lung and CCL21-Leu in the spleen or lung (Figure $3 \mathrm{~d}$ ). In addition, we cloned and sequenced the expressed CCL21 gene products in the spleen and lung by RT-PCR with primers that do not discriminate between the known CCL21 genes. Impressively, 11/11 clones in the spleen carried the serine at position 65 , whereas $4 / 4$ clones derived from the lung coded for leucine at position 65 , confirming the results from realtime PCR. Furthermore, in vivo administration of agonistic anti-LT $\beta R$, but not control antibodies, selectively stimulated the CCL21-Ser gene in both the spleen and lung (Figure 4a). The effects of LT $\beta R$ signaling were specific to CCL21-Ser, since CCL21-Leu transcriptional activity was not affected in the spleen and lung (Figure $4 a)$. These data indicate that LT selectively regulates CCL21-Ser but not CCL21-Leu.

Airway inflammation specifically induces CCL21-Ser in the lung in a LT-dependent fashion. To formally demonstrate that spleen and lung cells express the LT $\beta R$ and functionally respond to LT signals, we isolated splenic and lung stromal cells and tested their ability to respond to agonistic anti-LT $\beta$ R or hamster control antibodies. We found that both splenic and lung stromal cells responded to LT $\beta$ R signals by inducing the adhesion molecule VCAM-1 (Figure 4b). This induction was specific for the LT $\beta \mathrm{R}$, since $L T \beta R^{-/-}$ stromal cells, regardless of splenic or lung origin, did not respond to the anti-LT $\beta$ R antibody. Furthermore, CCL21 could be induced on embryonic fibroblasts 8 hours after stimulation with the agonistic antibody (data not shown). This demonstrates that spleen and lung cells not only express the LT $\beta$ R but are capable of responding to membrane LT signals. Therefore, we proposed that the paucity of LT-expressing cells in the lungs of unchallenged mice may not allow for strong stimulation of the CCL21-Ser gene.

To test our hypothesis, we sought to recruit LT expressing cells to the lung in a model of airway inflammation to further induce pulmonary CCL21 expression via CCL21-Ser. An S. mansoni SEA airway hypersensitivity model was chosen for its ability to induce a strong immune response in the lung. SEA challenge significantly induced pulmonary CCL21 expression, about two- to threefold above unchallenged mice ( $P=0.0005$ in the Student's $t$ test between naive and challenged groups) (Figure $4 \mathrm{c}$ ). To determine which of the CCL21 genes is responsible for the increased expression upon airway challenge, real-time PCR analysis was performed to distinguish between the two CCL21 genes. We found that an SEA challenge specifically induces the transcriptional activity of CCL21-Ser by approximately sixfold, whereas no significant induction was detected in CCL21-Len (Figure 4d). Since the activity of CCL21-Ser is regulated by LT in the spleen, we assessed the role of LT in pulmonary CCL21- 
Ser induction. To this aim, we used antagonistic antiLT $\beta$ or control antibodies during the challenge phase only in order to not disturb priming in our SEA model. This anti-LT $\beta$ treatment significantly reduced the levels of CCL21 from the control-challenged group $(P=$ 0.0009 between SEA-challenged and anti-LT $\beta$-treated groups). Impressively, blockade of membrane LT only in the challenge phase abolished the induction in CCL21 to preairway challenge levels $(P=0.51$ between naive and SEA-challenged anti-LT $\beta$ treated groups) (Figure 4c). As further support, SEA-challenged $L T \alpha^{-1}$ mice failed to induce CCL21 in the lung and had significantly lower levels of CCL21 than SEA-challenged WT mice $(P=0.013$ between SEA-challenged WT and $\left.L T \alpha^{-/}\right)$, confirming the requirement of $L T$ in pulmonary CCL21 induction (Figure 4c). These results demonstrate that chemokines such as CCL21 can be further induced in the lung under local inflammatory conditions and that this may serve to further stimulate the migration and homing of effector cells.

Homing of T cells to the lung requires CCR7 and CCL21. Although CCL21 has been shown to attract activated T cells in vitro, its role in vivo for primed $\mathrm{T}$ cells remains to be understood $(27,28,34)$. In order to ascribe a role for CCL21/CCR7 interactions in cell migration to the lung, we took advantage of the inflammatory leukocytes in the lungs of SEA-challenged mice for adoptive transfer to naive mice. We reasoned that because $T$ cells from the lungs of SEA-challenged mice have already homed to the lung, they should therefore possess all the homing receptors necessary for entry to the lung. The $T$ cells from SEAchallenged mice are predominantly $\mathrm{CD} 4^{+} \mathrm{Th} 2$ phenotype cells that have acquired an activated phenotype (CD62 $\mathrm{L}^{\text {low }}$ ) and express the CCR7 receptor (Figure 5a). In addition, the majority of the $T$ cells also stain $\mathrm{CD} 44^{\text {high }}$ (data not shown). Inflammatory leukocytes were isolated from lungs of SEA-challenged WT mice, fluorescently labeled with CFSE and Thy1.2-PE antibodies, treated with either PBS or a high dose of CCL19 to desensitize the CCR7 receptor prior and adoptively transferred to naive $L T \alpha^{-/}$recipient mice. $L T \alpha^{-/}$mice were used as recipients rather than WT mice, which have relatively higher expression of CCL21 in lymphoid tissues that may preferentially divert homing away from the lung. To rule out nonhoming-related effects, such as further differentiation or activation, and to avoid CCR7-resensitization, $L T \alpha^{/-}$recipient mice were killed 1 hour after transfer to determine the number of $\mathrm{T}$ cells that homed back to the lung. The mice were bled and perfused to eliminate leukocyte contamination from the circulation. $T$ cells (Thy $1.2^{+} \mathrm{CFSE}^{+}$) from the CCR7-desensitized group significantly decreased homing to the lung than control $\mathrm{T}$ cells, whereas non-T cells (Thy1.2-CFSE ${ }^{+}$) did not seem to be affected by CCR7 desensitization $(P<0.05$ between $\mathrm{T}$ cells from CCR7-desensitized and control groups) (Figure $5 \mathrm{~b}$ ). Furthermore, lung sections from the CCR7desensitized group exhibited approximately a threefold reduction in the number of Thy1-PE antibody-labeled cells compared with the control group and confirmed their localization to the lung tissues. These results demonstrate for the first time the importance of CCR7 on the $\mathrm{T}$ cell for homing to nonlymphoid tissues.

The foregoing results suggested that CCL21, a chemokine for CCR7 in the lung, mediates T cell homing to the lung. CCR7, however, binds to both CCL19 and CCL21. We did not believe that the effects of CCR7 desensitization were attributed to CCL19, since it was neither detectable by Northern blot (35) nor by sensitive ELISA (data not shown) in the lungs of the recipient mice, whereas CCL21 is readily detectable (Figure $3 a)$. To directly dissect the role of CCL21 in T cell homing to the lung, we pretreated the recipient mice with neutralizing anti-CCL21 or control antibodies prior to adoptive transfer of labeled inflammatory leukocytes from the lung. Neutralization of CCL21 dramatically decreased the number of $\mathrm{T}$ cells that homed to the lung after 4 hours ( $P=0.03$ between the two groups) (Figure $5 c)$. These results indicate that CCL21 is essential for $T$ cell homing to the lung.

\section{Discussion}

Chemokines are likely responsible for shifting the immune response from lymphoid tissues to nonlymphoid tissues $(8,15)$. CCL21 is expressed in both lymphoid and nonlymphoid tissues, but its role in peripheral nonlymphoid tissues in attracting lymphocytes has not been well defined. Despite cellular localization and homing defects in the spleen, LT-deficient mice have

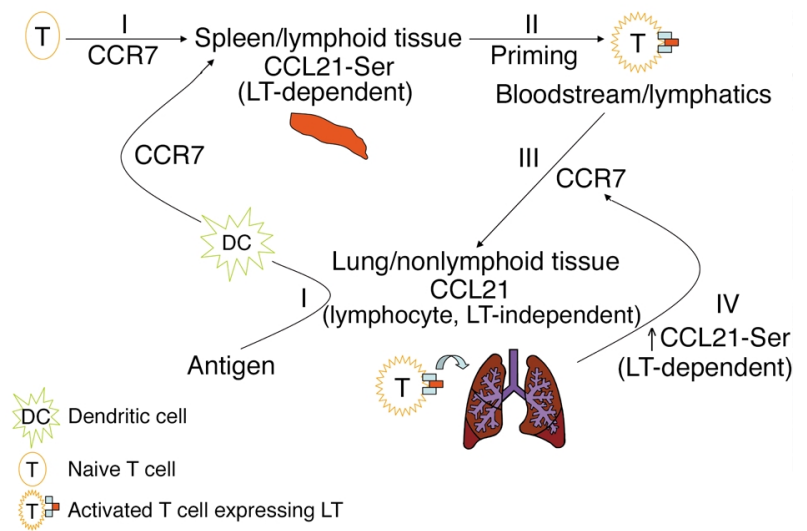

Figure 6

Model for the dual roles of CCL21 in central and peripheral immune responses. (I) Pathogen breaches and infects the lung/nonlymphoid tissue where antigen is picked up by DCs, which along with naive $T$ cells migrate to the draining lymph node/lymphoid tissue through CCL21-Ser/CCR7 interactions. (II) DCs prime antigen-specific T cells leading to their activation, proliferation, and differentiation. (III) The initial wave of primed $T$ cells enters the circulation and migrates to the lung directed by the constitutively expressed CCL21, which is independent of lymphocytes and LT. (IV) LT expressed by activated T cells in the lung stimulates the LT $\beta R$ on stromal cells, further inducing CCL21 expression via CCL21-Ser to attract additional T cells, resulting in a positive feedback loop. Additionally, the induced CCL21 may be localized to infected microregions within the lung to enhance targeting of effector cells to those infected areas. LT may also be derived from other inflammatory cells. 
increased accumulation of T cells, B cells, and DCs in the lungs, but the mechanism underlying this phenotype is unclear. In this study, we found that LT is required for the expression of CCL21 in the spleen but not in the lung. Our findings reveal genetic and functional specificities for the different CCL21 genes and, more importantly, a novel role for CCL21/CCR7 in the homing of $\mathrm{T}$ cells to the lung. Constitutively expressed CCL21 in the lung is LT and lymphocyte independent but appears to be important for initial recruitment. However, the introduction of inflammatory cells to nonlymphoid organs is sufficient to induce expression of the lymphoid CCL21-Ser in an LT-dependent fashion. This induction of CCL21-Ser may be part of a positive amplification loop to allow a rapid influx of lymphocytes from draining lymphoid tissues. The failure of $T$ cells to home to the lung in mice pretreated with neutralizing CCL21 antibodies or upon CCR7 desensitization further suggests an essential role for CCL21 in attracting primed $\mathrm{T}$ cells via CCR7 into the periphery. Collectively, these findings establish a novel and dual role for CCL21/CCR7 in regulating the balance between central and peripheral immune responses.

It is a widely held notion that a cell's responsiveness to chemokine gradients plays a major role in determining cell homing to organs and compartmentalization within those organs $(8,15)$. At first glance, the enhanced homing of T cells to lungs of LT-deficient mice seems to be at odds with this idea, since the absolute levels of CCL21 in the lung are comparable in WT and LT-deficient mice (Figures 1c and 3a). However, when one compares the relative levels of CCL21 between the spleen and lung of WT and LT-deficient mice, a key difference between the mice becomes apparent. The ratio of spleen-to-lung CCL21 is approximately 3:1 in WT mice. The loss of splenic CCL21 coupled with the retention of CCL21 in the lung flips the ratio to $1: 3$ in LT-deficient mice, thereby attracting more $T$ lymphocytes to the lung. This may be thought of as chemokine gradients acting across the entire mouse with numerous depots (some larger than others) of CCL21, each competing to draw its share of lymphocytes. Alternatively, CCL21 density may be thought of as a molecular sieve to net lymphocytes. For example, the high expression of CCL21 in WT spleens would represent a tightly woven net that would trap lymphocytes efficiently. In contrast, the LT-deficient spleen with its low expression of CCL21 would have a more porous net that allows lymphocytes to escape and become trapped by the finer CCL21 net in the lung. We believe it is the level of chemokines relative to other organs that determines the efficiency of homing.

Another possible explanation for the lymphoaccumulation in the lungs of LT-deficient mice is that they have opportunistic low-grade pulmonary infections that induce chemokines to draw lymphocytes to the inflamed tissue. Exogenous bacterial or viral challenge to the airways have shown that LT-deficient mice succumb more easily than their WT counterparts
(36-38). However, several points argue against this possibility: (a) the lung chemokine profiles between WT and $L T \alpha^{-1-}$ mice are strikingly similar (Figure $2 \mathrm{~b}$ ); (b) the ratio of naive to activated phenotype T cells are comparable in WT and $L T \alpha^{/-}$mice (Figure 1b); (c) we have observed consistent leukocytic accumulations in all of our LT-deficient mice instead of the patchy accumulations (high cellularity in some mice but not others) expected from opportunistic infections; (d) we have collected lung tissues from LT-deficient mice to assay for potential bacterial infections but found no bacterial colonies in our cultures; and (e) LT-deficient mice do not show lymphoaccumulation in the gut, another site of opportunistic infection. Consistently, we found diminished levels of CCL21 in the gut of LTdeficient mice (22).

Our model outlines the essential role of CCL21/ CCR7 interactions for T cell entry to nonlymphoid tissues (Figure 6). CCL21 is a major chemokine expressed in the lung that serves to attract $\mathrm{CCR}^{+}$cells, employing both LT-dependent and -independent pathways. Although CCL 21 is positively regulated by LT in lymphoid and nonlymphoid tissues, prior expression of CCL21 in nonlymphoid tissues is required for the initial wave of T cells to gain entry. LT signaling has been shown to play a critical role in peripheral tissues in the formation of lymphoidlike structures in situations of chronic inflammation $(39,40)$. Using rat insulin promoter-CCL21 (RIP-CCL21) and RIP-CXCL13 transgenic mice to ectopically express chemokines in the islets, Luther et al. found that the formation of lymphoid aggregates could be initially mediated by LTindependent chemokines and then expanded in a LTdependent fashion $(40,41)$. These elegant studies support our notion that LT-independent CCL21 in the lung may be important to initiate the recruitment of $\mathrm{T}$ cells to the lung while LT from activated T cells further stimulate CCL21-Ser to attract additional activated T cells into inflamed peripheral tissues. The apparent genetic redundancy in CCL21 gene duplication now becomes biologically relevant and resolves this issue via constitutive expression of the dominant CCL21-Len gene in nonlymphoid tissues. After recruitment of LTexpressing cells to the lung, LT $\beta$ R signaling on the resident lung cells promotes additional CCL21 expression (ratio of spleen:lung CCL21 changes from 3:1 for naive mice to 1.5:1 in airway-challenged mice). CCL21 induction on nonlymphoid tissues can serve at least two purposes: (a) to elevate the chemokine gradient for the recruitment of additional CCR $7^{+}$cells to the infected tissue, amplifying the inflammatory response in the fashion of a positive feedback loop or (b) to organize high local concentrations of CCL21 specifically targeted to the infected sites within the tissue for efficient guidance to the final effector site.

Although our study demonstrates a crucial role for CCR7 in Tlymphocyte homing to the lung, CCR7 is likely not the only molecule that is involved in the process. We do not exclude the possibility that other chemokines 
and adhesion molecules are intimately involved in trafficking to the lung. The presence of both activated and resting B cells as well as immature and mature DCs in the lungs suggests that both CCR7/CCL21-dependent and -independent mechanisms are involved under steady-state conditions. As CCR7 is expressed on most $\mathrm{T}$ lymphocytes, including naive and effector/memory cells, CCR7 is unlikely the chemokine receptor providing organ-specific homing to the lung $(29,42)$. This point is further supported by the relatively ubiquitous expression of CCL21, a major ligand for CCR7 $(27,43)$. CCR7/CCL21 may rather serve as coreceptors to facilitate homing to nonlymphoid tissues.

In this study, we have further identified a subset of chemokines that requires LT for its basal expression in the spleen as well as a subset that does not depend on LT for steady-state expression in the spleen. The lymphoid tissue chemokines CCL21, CCL19, and CXCL13 predominantly attract naive lymphocytes and are part of the first set of chemokines shown to be LT dependent (17). Interestingly, we found that the basal expression of CXC chemokine receptor ligand 10 (CXCL10), CCL5, and stromal cell-derived factor 2 (SDF-2) are also regulated by LT in the spleen. IP-10/CXCL10 and RANTES/CCL5, on the other hand, are involved in attracting activated $\mathrm{T}$ lymphocytes (44). The role of SDF-2, a chemokine originally cloned from the ST2 stromal cell line, is not known. (45). The significance of LT-mediated CXCL10, CCL5, and SDF-2 remains to be determined.

Are there other chemokines that are differentially regulated in lymphoid and nonlymphoid tissues? CXCL13 expression is LT-dependent in lymphoid tissues such as the spleen, $\mathrm{LN}$, and gut $(17,22)$. However, LT-deficient mice still retain WT levels of CXCL13 in the peritoneal cavity (46). This distinct regulation mechanism for CXCL13 in the peritoneal cavity allows for the homeostasis of $\mathrm{B} 1$ peritoneal cavity cells independent of LT, whereas B cell homeostasis is perturbed in the spleen and gut of LT-deficient mice $(22,46)$. Although only one CCL21 gene has been identified thus far in humans, it may also be subject to LTdependent and -independent control as the single CXCL13 gene is in mice. Thus the concept of differential regulation of chemokines in lymphoid and nonlymphoid tissues may be a more generalizable one that is not restricted to LT and CCL21. The differential regulation of chemokines with respect to tissue type may facilitate lymphocyte trafficking from lymphoid to nonlymphoid tissues (15). Overlapping and distinct chemokines between lymphoid and nonlymphoid tissues provide both familiar and new molecular cues to achieve efficiency and specificity. Our study, therefore, opens a new avenue in the exploration of molecular mechanisms for tissue-specific responses.

\section{Acknowledgments}

We thank Amanda Tesciuba and Anne Sperling for instruction on SEA challenges, Sumit Subudhi, James O'Keefe, and Shirley Bond for help with fluorescence microscopy, and Charles Lo for critical review of the manuscript. Anti-LT $\beta$, anti-LT $\beta R$ (for in vitro stimulation), and control hamster antibodies were generously provided by Jeff Browning (Biogen, Cambridge, Massachusetts, USA). ELC-Fc was a generous gift from Jason Cyster (University of California-San Francisco, San Francisco, California, USA). J.C. Lo and R.K. Chin are part of the Medical Scientist Training Program at the University of Chicago and supported by a Medical Scientist National Research Service Award (5 T32 GM07281). This research was in part supported by NIH grants HD-37104, DK-58897, and AI33068.

1. Fu, Y.X., and Chaplin, D.D. 1999. Development and maturation of secondary lymphoid tissues. Annu. Rev. Immunol. 17:399-433.

2. Ware, C.F., VanArsdale, T.L., Crowe, P.D., and Browning, J.L. 1995. The ligands and receptors of the lymphotoxin system. Curr. Topics Microbiol. Immunol. 198:175-218. (Review)

3. Karin, M., and Lin, A. 2002. NF-kappaB at the crossroads of life and death. Nat. Immunol. 3:221-227.

4. Alcamo, E., et al. 2002. Requirement for the NF-kappaB family member RelA in the development of secondary lymphoid organs. J. Exp. Med. 195:233-244.

5. Yin, L., et al. 2001. Defective lymphotoxin-beta receptor-induced NFkappaB transcriptional activity in NIK-deficient mice. Science. 291:2162-2165.

6. Franzoso, G., et al. 1998. Mice deficient in nuclear factor (NF)-kappa$\mathrm{B} / \mathrm{p} 52$ present with defects in humoral responses, germinal center reactions, and splenic microarchitecture. J. Exp. Med. 187:147-159.

7. Matsushima, A., et al. 2001. Essential role of nuclear factor (NF)-kappaB-inducing kinase and inhibitor of kappaB (IkappaB) kinase alpha in NF-kappaB activation through lymphotoxin beta receptor, but not through tumor necrosis factor receptor I. J. Exp. Med. 193:631-636.

8. Cyster,J.G. 1999. Chemokines and cell migration in secondary lymphoid organs. Science. 286:2098-2102.

9. Forster, R., et al. 1999. CCR7 coordinates the primary immune response by establishing functional microenvironments in secondary lymphoid organs. Cell. 99:23-33.

10. Gunn, M.D., et al. 1999. Mice lacking expression of secondary lymphoid organ chemokine have defects in lymphocyte homing and dendritic cell localization. J. Exp. Med. 189:451-460.

11. Okada, T., et al. 2002. Chemokine requirements for B cell entry to lymph nodes and Peyer's patches. J. Exp. Med. 196:65-75

12. Vassileva, G., et al. 1999. The reduced expression of 6 Ckine in the plt mouse results from the deletion of one of two 6Ckine genes. J. Exp. Med. 190:1183-1188.

13. Nakano, H., and Gunn, M.D. 2001. Gene duplications at the chemokine locus on mouse chromosome 4: multiple strain-specific haplotypes and the deletion of secondary lymphoid-organ chemokine and EBI-1 ligand chemokine genes in the plt mutation. J. Immunol. 166:361-369.

14. Chen, S.C., et al. 2002. Ectopic expression of the murine chemokines CCL21a and CCL21b induces the formation of lymph node-like structures in pancreas, but not skin, of transgenic mice. J. Immunol. 168:1001-1008

15. Kunkel, E.J., and Butcher, E.C. 2002. Chemokines and the tissue-specific migration of lymphocytes. Immunity. 16:1-4.

16. Blease, K., Lukacs, N.W., Hogaboam, C.M., and Kunkel, S.L. 2000. Chemokines and their role in airway hyper-reactivity. Respir. Res. 1:54-61.

17. Ngo, V.N., et al. 1999. Lymphotoxin alpha/beta and tumor necrosis factor are required for stromal cell expression of homing chemokines in B and T cell areas of the spleen. J. Exp. Med. 189:403-412.

18. Wu, Q., et al. 1999. The requirement of membrane lymphotoxin for the presence of dendritic cells in lymphoid tissues. J. Exp. Med. 190:629-638.

19. Yu, P., et al. 2002. B cells control the migration of a subset of dendritic cells into B cell follicles via CXC chemokine ligand 13 in a lymphotoxindependent fashion. J. Immunol. 168:5117-5123.

20. De Togni, P., et al. 1994. Abnormal development of peripheral lymphoid organs in mice deficient in lymphotoxin. Science. 264:703-707.

21. Futterer, A., Mink, K., Luz, A., Kosco-Vilbois, M.H., and Pfeffer, K. 1998. The lymphotoxin $\beta$ receptor controls organogenesis and affinity maturation in peripheral lymphoid tissues. Immunity. 9:59-70.

22. Kang, H.S., et al. 2002. Signaling via LTbetaR on the lamina propria stromal cells of the gut is required for IgA production. Nat. Immunol. 3:576-582.

23. Tesciuba, A.G., et al. 2001. Inducible costimulator regulates Th2-mediated inflammation, but not Th2 differentiation, in a model of allergic airway disease. J. Immunol. 167:1996-2003. 
24. Guo, Z., et al. 2001. Cutting edge: membrane lymphotoxin regulates CD8(+) $\mathrm{T}$ cell-mediated intestinal allograft rejection. J. Immunol. 167:4796-4800.

25. Dejardin, E., et al. 2002. The lymphotoxin-beta receptor induces different patterns of gene expression via two NF-kappaB pathways. Immunity. 17:525-535.

26. Banks, T.A., et al. 1995. Lymphotoxin-alpha-deficient mice: effects on secondary lymphoid organ development and humoral immune responsiveness. J. Immunol. 155:1685-1693.

27. Gunn, M.D., et al. 1998. A chemokine expressed in lymphoid high endothelial venules promotes the adhesion and chemotaxis of naive T lymphocytes. Proc. Natl. Acad. Sci. U. S. A. 95:258-263.

28. Campbell, J.J., et al. 1998. 6-C-kine (SLC), a lymphocyte adhesion-triggering chemokine expressed by high endothelium, is an agonist for the MIP-3beta receptor CCR7. J. Cell Biol. 141:1053-1059.

29. Nagira, M., et al. 1998. A lymphocyte-specific CC chemokine, secondary lymphoid tissue chemokine (SLC), is a highly efficient chemoattractant for B cells and activated T cells. Eur. J. Immunol. 28:1516-1523.

30. Chan, V.W., et al. 1999. Secondary lymphoid-tissue chemokine (SLC) is chemotactic for mature dendritic cells. Blood. 93:3610-3616.

31. Kellermann, S.A., Hudak, S., Oldham, E.R., Liu, Y.J., and McEvoy, L.M. 1999. The CC chemokine receptor-7 ligands 6 Ckine and macrophage inflammatory protein-3beta are potent chemoattractants for in vitroand in vivo- derived dendritic cells. J. Immunol. 162:3859-3864.

32. Itakura, M., et al. 2001. Blockade of secondary lymphoid tissue chemokine exacerbates Propionibacterium acnes-induced acute lung inflammation. J. Immunol. 166:2071-2079.

33. Mori, S., et al. 2001. Mice lacking expression of the chemokines CCL21ser and CCL19 (plt mice) demonstrate delayed but enhanced T cell immune responses. J. Exp. Med. 193:207-218.

34. Willimann, K., et al. 1998. The chemokine SLC is expressed in T cell areas of lymph nodes and mucosal lymphoid tissues and attracts activated T cells via CCR7. Eur. J. Immunol. 28:2025-2034.

35. Ngo, V.N., Tang, H.L., and Cyster, J.G. 1998. Epstein-Barr virus-induced molecule 1 ligand chemokine is expressed by dendritic cells in lymphoid tissues and strongly attracts naive T cells and activated B cells. J. Exp. Med. 188:181-191.

36. Roach, D.R., et al. 2001. Secreted lymphotoxin-alpha is essential for the control of an intracellular bacterial infection. J. Exp. Med. 193:239-246.

37. Lund, F.E., et al. 2002. Lymphotoxin-alpha-deficient mice make delayed, but effective, $\mathrm{T}$ and $\mathrm{B}$ cell responses to influenza. J. Immunol. 169:5236-5243.

38. Ehlers, S., et al. 2003. The lymphotoxin beta receptor is critically involved in controlling infections with the intracellular pathogens mycobacterium tuberculosis and Listeria monocytogenes. J. Immunol. 170:5210-5218.

39. Wu, Q., et al. 2001. Reversal of spontaneous autoimmune insulitis in nonobese diabetic mice by soluble lymphotoxin receptor. J. Exp. Med. 193:1327-1332.

40. Luther, S.A., et al. 2002. Differing activities of homeostatic chemokines CCL19, CCL21, and CXCL12 in lymphocyte and dendritic cell recruitment and lymphoid neogenesis. J. Immunol. 169:424-433.

41. Luther, S.A., Lopez, T., Bai, W., Hanahan, D., and Cyster, J.G. 2000. BLC expression in pancreatic islets causes B cell recruitment and lymphotoxin-dependent lymphoid neogenesis. Immunity. 12:471-481.

42. Sallusto, F., et al. 1999. Switch in chemokine receptor expression upon TCR stimulation reveals novel homing potential for recently activated T cells. Eur. J. Immunol. 29:2037-2045.

43. Nagira, M., et al. 1997. Molecular cloning of a novel human CC chemokine secondary lymphoid-tissue chemokine that is a potent chemoattractant for lymphocytes and mapped to chromosome $9 \mathrm{p} 13$. J. Biol. Chem. 272:19518-19524.

44. Lukacs, N.W. 2000. Migration of helper T-lymphocyte subsets into inflamed tissues. J. Allergy Clin. Immunol. 106(Suppl.):S264-S269. (Review)

45. Hamada, T., et al. 1996. Isolation and characterization of a novel secretory protein, stromal cell-derived factor-2 (SDF-2) using the signal sequence trap method. Gene. 176:211-214.

46. Ansel, K.M., Harris, R.B., and Cyster, J.G. 2002. CXCL13 is required for B1 cell homing, natural antibody production, and body cavity immunity. Immunity. 16:67-76. 